\title{
Excitonic photoluminescence linewidths in AlGaAs grown by molecular beam epitaxy
}

\author{
D. C. Reynolds, K. K. Bajaj, and C. W. Litton \\ Electronic Research Branch, AFWAL/AADR, Wright-Patterson AFB, Ohio 45433 \\ P. W. Yu \\ University Research Center, Wright State University, Dayton, Ohio 45345 \\ J. Klem, C. K. Peng, and H. Morkoç \\ Department of Electrical Engineering and Coordinated Science Laboratory, I101 West Springfield Avenue, \\ University of Illinois, Urbana, Illinois 61801 \\ Jasprit Singh \\ Department of Electrical Engineering and Computer Science, University of Michigan, Ann Arbor, \\ Michigan 48109
}

(Received 27 November 1985; accepted for publication 20 January 1986)

\begin{abstract}
The linewidths of excitonic transitions were measured in $\mathrm{Al}_{x} \mathrm{Ga}_{1-x} \mathrm{As}$, grown by molecular beam epitaxy as a function of alloy composition $x$ for values of $x \leqslant 0.43$ using high resolution photoluminescence spectroscopy at liquid helium temperature. The values of the linewidths thus measured are compared with the results of several theoretical calculations in which the dominant broadening mechanism is assumed to be the statistical potential fluctuations caused by the components of the alloy. An increase in the linewidth as a function of $x$ is observed which is in essential agreement with the prediction of the various theoretical calculations. The linewidths of the excitonic transitions in $\mathrm{Al}_{x} \mathrm{Ga}_{1-x}$ As observed in the present work are the narrowest ever reported in the literature, for example $\sigma=2.1 \mathrm{meV}$ for $x=0.36$, thus indicating very high quality material
\end{abstract}

Recent advances in epitaxial crystal growth techniques such as molecular beam epitaxy (MBE) and metalorganic chemical vapor deposition (MOCVD) have allowed the fabrication of a variety of optoelectronic and microwave devices, many of which have a semiconductor alloy as an important constituent. One of the most useful techniques for obtaining information concerning the quality of the alloys is photoluminescence spectroscopy. The shape of the excitonic transition contains a great deal of information about the quality of the alloy. Generally, the excitonic emission spectra in semiconductor alloys are considerably broader than those observed in their binary components. This broadening has been attributed to compositional disorder present in alloys. An alloy can be random on an atomic scale or on a scale $r_{c}$ in which case $r_{\mathrm{c}}$ defines the radius of the clusters in the alloys. Goede et al. ${ }^{1}$ were the first to calculate $\sigma$, the full width at half-maximum (FWHM) of the excitonic lines as a function of alloy composition in perfectly random alloys. Subsequently, Singh and Bajaj ${ }^{2}$ and Schubert et al. ${ }^{3}$ not being aware of the work of Goede et al., ${ }^{1}$ independently calculated the variation of $\sigma$ as a function of alloy composition treating the exciton as a classical system. These two groups arrived at the same result. The values of $\sigma$ these two groups calculate are, however, about 2.7 times larger than those calculated by Goede et al. ${ }^{1}$ Recently Singh and Bajaj ${ }^{4}$ have calculated the variation of $\sigma$ as a function of alloy composition following a quantum mechanical approach.

All of the $\mathrm{Al}_{x} \mathrm{Ga}_{1-x}$ As samples used in this study were grown in a MBE system having very low background impurity contamination, achieved predominantly through the use of extensive cryopaneling within the growth chamber. Samples were grown on (100) oriented semi-insulating GaAs substrates, $\mathrm{Cr}$-doped as well as undoped liquid encapsulated Czochralski wafers. Polished substrate wafers were cleaned, etched, and otherwise prepared for growth using techniques which have been described in detail elsewhere. ${ }^{5}$ Growth rates of approximately $1 \mu \mathrm{m} / \mathrm{h}$ yielded samples whose thicknesses varied between 1 and $2 \mu \mathrm{m}$. The highest quality $\mathrm{Al}_{x} \mathrm{Ga}_{1-x} \mathrm{As}$ samples were achieved at substrate temperatures in the range of $680-700^{\circ} \mathrm{C}$ with V/III flux ratios in the range of $6-10 / 1$. During growth the substrates were azimuthally rotated in the incident beam flux at rates of 3-5 rpm. A special two-zone cracking furnace was employed to produce the predominantly $\mathrm{As}_{2}$ flux which was used in the growth of these layers.

The optical transitions were analyzed by high resolution photoluminescence using a modified 4-m Bausch \& Lomb grating spectrograph. These photoluminescent measurement techniques have been described elsewhere. ${ }^{6}$ In the current study we have analyzed the photoluminescence spectra of $\mathrm{Al}_{x} \mathrm{Ga}_{1-x}$ As samples with values of $x$ varying from 0.13 to 0.43 . In Fig. 1 we display the optical transition observed in a sample with $x=0.15$. We assume that the highest energy transition at $1.6964 \mathrm{eV}$ is associated with the collapse of the free exciton. Adding the free-exciton binding energy of 7.9 $\mathrm{meV}$ from Pearah et al. ${ }^{7}$ to $1.6964 \mathrm{eV}$ we get the value of the band gap of $\mathrm{Al}_{x} \mathrm{Ga}_{1-x}$ As. Using the following relation":

$$
E_{g}(\mathrm{GaAlAs})=E_{g}(\mathrm{GaAs})+1.245 x
$$

we derive the value of $x=0.15$. The linewidth FWHM of the transition at $1.6890 \mathrm{eV}$ is determined to be $1.6 \mathrm{meV}$. This transition is most likely a free to bound transition (free hole to bound electron). This is a very sharp transition for an 


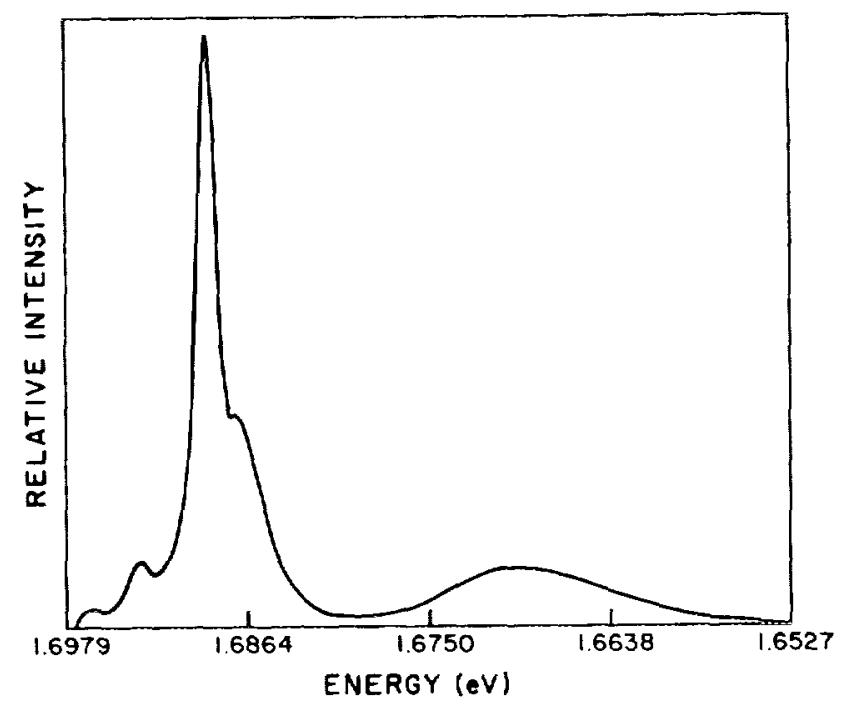

FIG. 1. Densitometer trace of the optical transitions in a sample with $x=0.15$. The highest energy transition is the free-exciton transition. The transition with the greatest intensity is most likely a free to bound transition and has a linewidth (FWHM) of $1.6 \mathrm{meV}$.

alloy composition of $x=0.15$. The optical transition for a sample with $x=0.36$ is shown in Fig. 2. This sample shows only one transition and that is assumed to be the free-exciton transition. Again taking the free-exciton binding energy of $25 \mathrm{meV}$ from Ref. 7 an $x$ value of 0.36 is determined. The linewidth (FWHM) for this line is $2.1 \mathrm{meV}$ which is also a very sharp line for an alloy composition of $x=0.36$. In a previous paper ${ }^{9}$ the excitonic transitions in the $\mathrm{Al}_{x} \mathrm{Ga}_{1-x}$ As alloy were traced back to the analogous transitions in GaAs for $x=0-0.13$. It was also reported that the excitonic transitions observed in different samples of $\mathrm{Al}_{x} \mathrm{Ga}_{1}$ As with roughly the same $x$ value showed considerable variation in linewidth. These same trends have been observed in the higher $x$-value region reported in this letter. The theory predicts that the linewidth will increase monotonically with $x$ value for a perfectly random alloy, here $r_{c}$ takes on the value of $2.21 \AA$. ${ }^{2}$ The theory also predicts that the linewidth depends on $r_{c}$ in the alloy. If there is any clustering of the $\mathrm{Al}$ atoms present in the material, the value of $r_{c}$ will be larger than $2.21 \AA$ and will lead to substantially larger values of the linewidth. Another important broadening mechanism at low

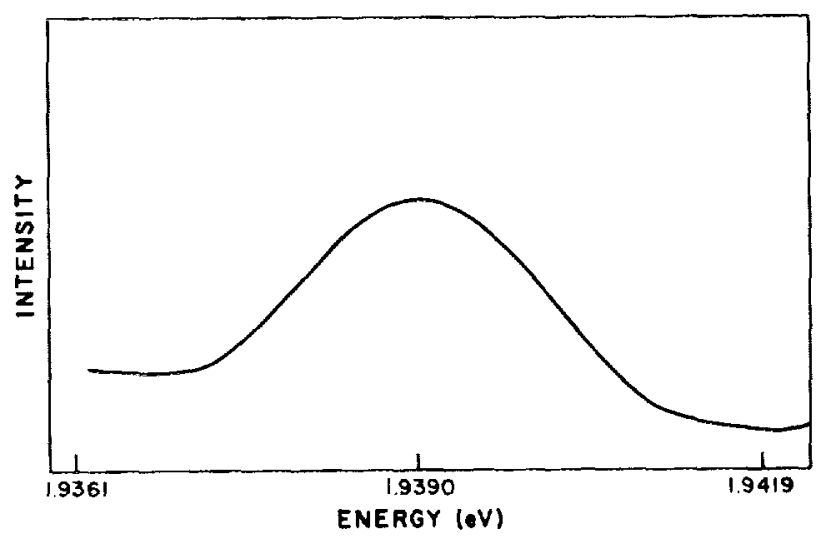

FIG. 2. Densitometer trace of the free-exciton transition for a sample with $x=0.36$. The linewidth (FWHM) of this transition is $2.1 \mathrm{meV}$. temperatures is due to electric fields produced by the random distribution of ionized impurities. ${ }^{10}$ In high quality alloys the ionized impurity concentration will usually be $<10^{16} / \mathrm{cm}^{3}$. At these concentrations this mechanism leads to linewidths $<1 \mathrm{meV}$. The dominant broadening mechanism therefore is alloy scattering with the sharpest lines being observed for completely random alloys. Clustering is then responsible for the linewidth variation observed for samples having the same $x$ value.

In Fig. 3 we have plotted the values of the width of the narrowest observed lines in $\mathrm{Al}_{x} \mathrm{Ga}_{1-x}$ As for several values of $x$ (open squares) along with the variation of $\sigma$ as calculated by several groups assuming a perfectly random alloy. The dashed curve is obtained by using the expressions of Singh and $\mathrm{Bajaj}^{2}$ and Schubert et al., ${ }^{3}$ treating the exciton as a classical system of volume $V_{\mathrm{ex}}=(4 \pi / 3) R_{\mathrm{ex}}^{3}$ where $R_{\mathrm{ex}}$ is an exciton radius. Recently, Singh and Bajaj ${ }^{4}$ have calculated $\sigma$ as a function of $x$ following a quantum mechanical approach. The solid curve represents their results which are about a factor 3 smaller than those obtained in Refs. 2 and 3. Goede et al., in their calculation, use for $V_{\text {ex }}$ an expression $(4 \pi / 3)\left\langle r^{3}\right\rangle$, where $\left\langle r^{3}\right\rangle$ is an expectation value of $r^{3}$ and is equal to $7.5 R_{\mathrm{ex}}^{3}$. As $\sigma$ varies as $\left(V_{\mathrm{ex}}\right)^{1 / 2}$ (Ref. 2) the linewidths calculated in Refs. 2 and 3 are about a factor 2.7 larger than those calculated by Goede $e t$ al. ${ }^{1}$ The values of $\sigma$ calculated by Singh and Bajaj following a quantum mechanical approach are about $10 \%$ smaller than those of Goede et al. ${ }^{1}$ Also shown in Fig. 3 are some experimental values of $\sigma$ from the literature. ${ }^{11-14}$ We point out that the experimental values of $\sigma$ reported by Schubert et al. ${ }^{3}$ in MBE grown $\mathrm{Al}_{x} \mathrm{Ga}_{1-x}$ As with $x$ values varying from 0.29 to 0.38

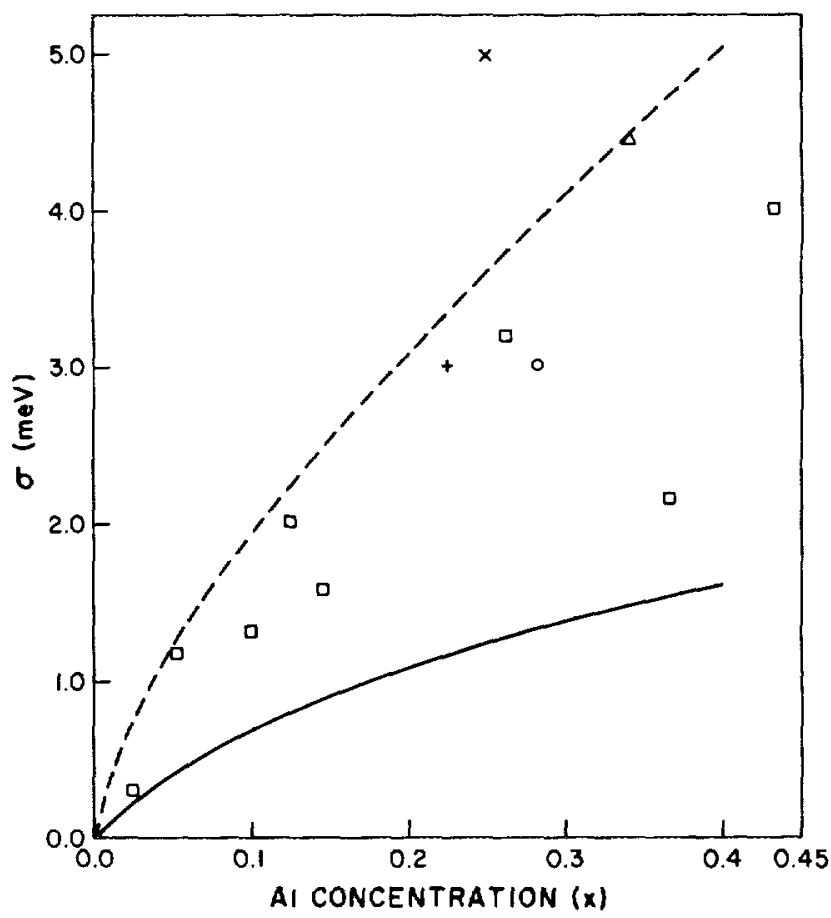

FIG. 3. Variation of $\sigma$ as a function of $x$ up to $x=0.43$. The experimental points are $(\square)$ (current experiment), $(+)$ (see Ref. 11), (O) (see Ref. 12), ( $\triangle$ ) (see Ref. 13), (X) (see Ref. 14). Dashed and solid curves are theoretical curves. 
are much larger than those observed in this study (e.g., 5.2 $\mathrm{meV}$ for $x=0.29$ and $8.2 \mathrm{meV}$ for $x=0.38$ ).

In conclusion, we have measured the excitonic photoluminescence linewidth in MBE grown $\mathrm{Al}_{x} \mathrm{Ga}_{1-x}$ As as a function of alloy composition. We find that the observed linewidths are somewhat larger than those calculated using a quantum mechanical approach assuming a perfectly random alloy thus suggesting a possible improvement in the quality of AlGaAs in the future. The linewidths we observe are the narrowest ever reported in AlGaAs reflecting very high quality material.

The work at the University of Illinois was sponsored by the Air Force Office of Scientific Research.

'O. Goede, L. John, and D. Henning, Phys. Status Solidi B 89, K183 (1978).

${ }^{2}$ J. Singh and K. K. Bajaj, Appl. Phys. Lett. 44, 1975 (1984).
${ }^{3}$ E. F. Schubert, E. O. Gobel, Y. Horikoshi, K. Ploog, and H. J. Queisser, Phys. Rev. B 30, 813 (1984).

${ }^{4}$ D. C. Reynolds, C. W. Litton, K. K. Bajaj, P. W. Yu, J. Singh, P. J. Pearah, W. T. Masselink, T. Henderson, J. Klem, and H. Morkoç, J. Vac. Sci. Technol. B (in press).

${ }^{5}$ T. J. Drummond, H. Morkoç, and A. Y. Cho, J. Cryst. Growth 56, 449 (1982).

'D. C. Reynolds, K. K. Bajaj, C. W. Litton, and E. B. Smith, Phys. Rev. B 28, $3300(1983)$

${ }^{7}$ P. J. Pearah, W. T. Masselink, J. Klem, T. Henderson, H. Morkoç, C. W. Litton, and D. C. Reynolds, Phys. Rev. B 32, 3857 (1985).

${ }^{8}$ H. C. Casey and M. B. Panish, Heterostructure Lasers (Academic, New York, 1978), Part A, Chap. 4

${ }^{9}$ D. C. Reynolds, K. K. Bajaj, C. W. Litton, J. Singh, P. W. Yu, T. Henderson, P. Pearah, and H. Morkoç, J. Appl. Phys. 58, 1643 (1985)

${ }^{10}$ D. M. Larsen, Phys. Rev. B 8, 535 (1973).

"G. Wicks, W. I. Wang, C. E. C. Wood, L. F. Eastman, and L. Rathbun, J. Appl. Phys. 52, 5792 (1981).

12J. M. Ballingall and D. M. Collins, J. Appl. Phys. 54, 34 (1983).

${ }^{13}$ M. Heiblum, E. E. Mendez, and L. Osterling, J. Appl. Phys. 54, 6982 (1983).

${ }^{14}$ J. R. Shealy, V. H. Kreismanis, D. K. Wagner, and J. M. Woodall, Appl. Phys. Lett. 42, 83 (1983). 